\title{
Geometric approach to nuclear pasta phases
}

\author{
Sebastian Kubis ${ }^{*}$ and Włodzimierz Wójcik \\ Cracow University of Technology, Institute of Physics, Podchorażych 1, 30-084 Kraków, Poland
}

(Received 14 October 2016; published 20 December 2016)

\begin{abstract}
By use of the variational methods and differential geometry in the framework of the liquid drop model we formulate appropriate equilibrium equations for pasta phases with imposed periodicity. The extension of the Young-Laplace equation in the case of charged fluid is obtained. The $\beta$ equilibrium and virial theorem are also generalized. All equations are shown in gauge invariant form. For the first time, the pasta shape stability analysis is carried out. The proper stability condition in the form of the generalized Jacobi equation is derived. The presented formalism is tested on some particular cases.
\end{abstract}

DOI: 10.1103/PhysRevC.94.065805

\section{INTRODUCTION}

The crust-core transition region in a neutron star represents a place where the lattice of neutron-rich nuclei must have been replaced by the homogeneous npe matter. On the bulk level, the thermodynamical considerations allow to determine the density where the homogeneous phase is unstable against the charge density perturbation and the two-phase system is formed [1]. The two phases have different charges and they are separated by the phase boundary with nonzero surface tension. The competition between the surface and Coulomb energy leads to a formation of structures with various shapes, dependent on the average density of the system. In this way, the infinitely long structures, usually called pasta phases, are formed and interpolate between the homogeneous matter and the lattice of spherical nuclei. On the scale larger than the size of those structures the npe matter is neutral, so it is convenient to introduce a Wigner-Seitz (WS) cell containing the two phases in thermodynamic equilibrium with different charges to ensure the neutrality of the cell. In principle, the WS cells should be periodically placed in space, which imposes the periodic boundary condition on it. However, for most of the applications and because of the simplicity, the periodicity is abandoned and the WS cells are represented by the isolated sphere, cylinder, or slab leading to names referring to Italian cooking like: gnocchi, spaghetti, and lasagna phases. Such a scheme was proposed in [2,3] and subsequently developed by many authors. The simplified geometry allows easily to determine the energy minimum for the WS cell with respect to the size of the cell and the size of the cluster. However, such simplification does not allow to examine the stability of such structures and find properties of the lattice which they should form. Some remarks on stability of proton spherical clusters in neutron gas were presented in [4]. However, one must note that a true analytic stability analysis of pasta phases was never done. In the work [5] structures taking shape of periodic labyrinths (gyroid, double gyroid, diamond-like) were explored in the framework of the liquid drop model. Introduction of such structures was motivated by their presence in block copolymers. The existence of pastas with different

*skubis@pk.edu.pl shapes with included periodicity was shown in terms of other approaches like the Thomas-Fermi approximation [6], Hartree-Fock calculations [7], or molecular dynamics [8].

The aim of this work is to analyze the existence of the pasta phase shapes by geometrical methods in a more rigorous way than it was done previously. We start here from the compressible liquid drop model (CLDM) that means the proton clusters are immersed in the electron-neutron gas in thermodynamic equilibrium. We do not make any assumptions on the cluster shape except the periodic boundary conditions required for the correct description of such system. In Sec. II we derive the equations governing the pasta's shape and show the relations between the various physical quantities like chemical potentials, pressure, etc. In Sec. III the stability considerations with respect to the cluster shape were carried out.

\section{VARIATIONAL ANALYSIS}

Our two phase system consists of the proton $(p+n+e)$ and neutron $(n+e)$ phase. On the phase boundary the surface tension $\sigma$ is present and it leads to formation of a proton cluster $\mathcal{P}$, see Fig. 1. The charge density of the proton cluster is positive $\rho_{+}=e\left(n_{p}-n_{e}\right)$, whereas in the neutron phase $\mathcal{N}$ is negative $\rho_{-}=-e n_{e}$, where $n_{i}$ is the number density of $i$ particle. When the proton clusters form a periodic lattice (one, two, and three dimensional) it is convenient to introduce a unit cell $\mathcal{C}$ obeying periodic boundary conditions. In general the unit cell is a parallelepiped. For illustrative purposes, we refer to the rectangular unit cell (cuboid), but we must emphasize that the whole further reasoning is also valid for the nonrectangular unit cell-the only property which is essential is the periodicity of physical quantities describing the two phases. In our analysis we do refer neither to the primitive cell (by definition containing only one lattice point) nor to the Wigner-Seitz cell which is a specific case of the primitive cell. We would like to note that WS cell, defined by the locus of points that are closer to a given lattice point than to any of the other lattice points, should not be confused with the so-called Wigner-Seitz approximation which is based on the isolated cell devoid periodicity. By its construction, the WS cell for typical 3D lattices like $f c c$, $b c c$, or 2D lattices such as the triangular one, takes the form of complicated polyhedra. However, all of these typical lattices occurring in the pasta 


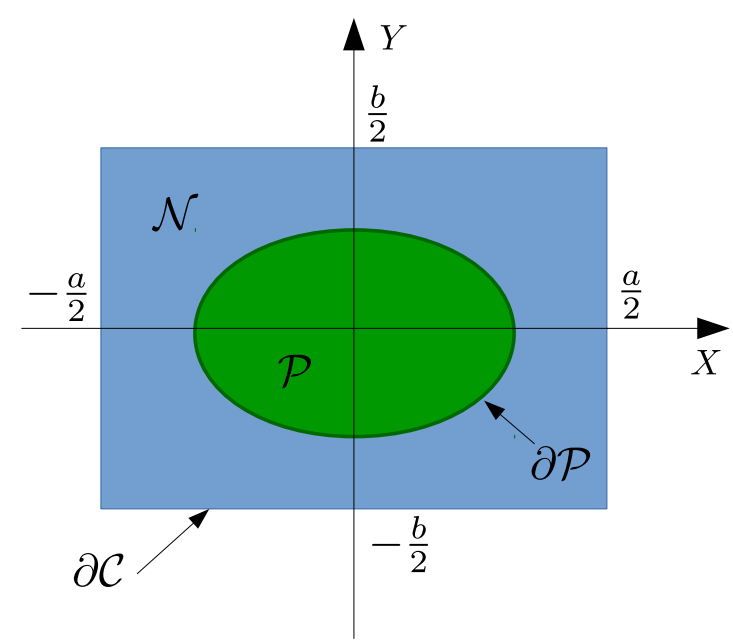

FIG. 1. Schematic view of rectangular cell with one proton cluster $\mathcal{P}$. The surface tension is associated with its boundary $\partial \mathcal{P}$.

phases can be described by the use of rectangular unit cells with a higher number of lattice points (proton clusters).

The charge separation between the phases generates the electrostatic potential $\Phi$. For cuboid cell $a \times b \times c$ the periodicity of the electrostatic potential $\Phi(\boldsymbol{x})$ is expressed by the following conditions:

$$
\begin{aligned}
& \Phi\left(-\frac{a}{2}, y, z\right)=\Phi\left(\frac{a}{2}, y, z\right), \\
& \Phi\left(x,-\frac{b}{2}, z\right)=\Phi\left(x, \frac{b}{2}, z\right), \\
& \Phi\left(x, y,-\frac{c}{2}\right)=\Phi\left(x, y, \frac{c}{2}\right) .
\end{aligned}
$$

Proton cluster $\mathcal{P}$ occupies volume $V_{\mathcal{P}}$ whereas the cell has volume $V_{\mathcal{C}}=a b c$. The cell is neutral as a whole, which means $N_{p}=N_{e}$ or for particle densities

$$
\frac{V_{\mathcal{P}}}{V_{\mathcal{C}}} n_{p}-n_{e}=0 .
$$

In the $\mathcal{P}$ phase, both nucleon number densities $n_{p}$ and $n_{n}^{\mathcal{P}}$ do not vanish, while in the $\mathcal{N}$ phase only the neutron density $n_{n}^{\mathcal{N}}$ is nonzero. Electrons are treated as being homogeneously distributed in the whole cell, because their screening length is much larger than the cell size [1]. On the scales larger than the cell size, the matter has an average baryon number density $\bar{n}$. The baryon number conservation leads to the relation between those densities:

$$
\bar{n}=\frac{V_{\mathcal{P}}}{V_{\mathcal{C}}}\left(n_{p}+n_{n}^{\mathcal{P}}\right)+\left(1-\frac{V_{\mathcal{P}}}{V_{\mathcal{C}}}\right) n_{n}^{\mathcal{N}} .
$$

The total energy density for an individual cell is a function of particle densities in both phases, the shape of the cluster surface $\partial \mathcal{P}$, and cell sizes:

$$
\begin{aligned}
\varepsilon\left(n_{p}, n_{n}^{\mathcal{P}}, n_{n}^{\mathcal{N}}, n_{e}, \partial \mathcal{P}, a, b, c\right) \\
=\frac{V_{\mathcal{P}}}{V_{\mathcal{C}}} \varepsilon_{n u c}\left(n_{p}, n_{n}^{\mathcal{P}}\right)+\left(1-\frac{V_{\mathcal{P}}}{V_{\mathcal{C}}}\right) \varepsilon_{n u c}\left(0, n_{n}^{\mathcal{N}}\right) \\
\quad+\varepsilon_{e}\left(n_{e}\right)+\varepsilon_{S}+\varepsilon_{\text {Coul }},
\end{aligned}
$$

where the surface energy density is determined by the nonzero surface tension $\sigma$,

$$
\varepsilon_{S}=\frac{\sigma}{V_{\mathcal{C}}} \int_{\partial \mathcal{P}} d S,
$$

and the Coulomb energy density is

$$
\varepsilon_{\text {Coul }}=\frac{1}{2 V_{\mathcal{C}}} \int_{\mathcal{C}} \Phi(\boldsymbol{x}) \rho(\boldsymbol{x}) d^{3} x .
$$

The charge density distribution $\rho(\boldsymbol{x})$ takes the step function form

$$
\rho(\boldsymbol{x})= \begin{cases}\rho_{+}=e\left(n_{p}-n_{e}\right) & \text { for } x \in \mathcal{P} \\ \rho_{-}=-e n_{e} & \text { for } x \in \mathcal{N}\end{cases}
$$

and the electrostatic potential is solution of the Poisson equation with periodic boundary condition for the charge density given by Eq. (7):

$$
\nabla^{2} \Phi(\boldsymbol{x})=-4 \pi \rho(\boldsymbol{x}) .
$$

In the thermodynamic equilibrium the energy takes minimum with respect to the eight variables, see Eq. (4) under the two constraints: the first one coming from the neutrality of the individual cell Eq. (2) and the second one from the baryon number conservation Eq. (3). The convenient way is to make use of Lagrange multipliers

$$
\begin{aligned}
\tilde{\varepsilon}= & \varepsilon+\lambda_{Q}\left(\frac{V_{\mathcal{P}}}{V_{\mathcal{C}}} n_{p}-n_{e}\right) \\
& +\lambda_{B}\left(\bar{n}-\frac{V_{\mathcal{P}}}{V_{\mathcal{C}}}\left(n_{p}+n_{n}^{\mathcal{P}}\right)-\left(1-\frac{V_{\mathcal{P}}}{V_{\mathcal{C}}}\right) n_{n}^{\mathcal{N}}\right) .
\end{aligned}
$$

Minimization against the neutral particle densities $n_{n}^{\mathcal{N}}, n_{n}^{\mathcal{P}}$ corresponds to the vanishing of partial derivatives $\frac{\partial \tilde{\varepsilon}}{\partial n_{n}^{N}}$ and $\frac{\partial \tilde{\varepsilon}}{\partial n_{n}^{p}}$ which lead to the Gibbs conditions for neutron chemical potentials in both phases

$$
\lambda_{B}=\mu_{n}^{\mathcal{P}}=\mu_{n}^{\mathcal{N}} \equiv \mu_{n},
$$

where the chemical potentials of the neutron are the derivative of nuclear contribution to the total energy density $\frac{\partial \varepsilon_{n u c}\left(n_{p}, n_{n}\right)}{\partial n_{n}}$ taken at the appropriate phase densities. For further convenience we use only one $\mu_{n}$.

Any variation of charged particles density $\left(n_{e}, n_{p}\right)$ and proton cluster shape $\partial \mathcal{P}$ will make a variation of the potential $\delta \Phi$ and requires more attention. Potential variation $\delta \Phi$ is linearly dependent on the charge variation $\delta \rho$ by the Poisson equation

$$
\nabla^{2} \delta \Phi(\boldsymbol{x})=-4 \pi \delta \rho(\boldsymbol{x})
$$

Just as $\Phi$, the perturbation $\delta \Phi$ obeys periodic boundary conditions of type Eq. (1). It may be expressed by the Green function

$$
\delta \Phi(\boldsymbol{x})=\int_{\mathcal{C}} G_{P}\left(\boldsymbol{x}, \boldsymbol{x}^{\prime}\right) \delta \rho\left(\boldsymbol{x}^{\prime}\right) d^{3} x^{\prime}
$$


The Green function must obey periodic boundary conditions. For instance, in the case of the cuboid cell it takes the form [9]

$$
G_{P}\left(\boldsymbol{x}, \boldsymbol{x}^{\prime}\right)=\frac{4 \pi}{a b c} \sum_{m, n, k=-\infty}^{+\infty} \frac{\exp \left(i \boldsymbol{K}_{m n k} \cdot\left(\boldsymbol{x}-\boldsymbol{x}^{\prime}\right)\right)}{K_{m n k}^{2}}
$$

where the vector $\boldsymbol{K}_{m n k}=2 \pi\left(\frac{n}{a}, \frac{m}{b}, \frac{k}{c}\right)$ and the prime sign means that the summation excludes the $m=n=k=0$ term. For a nonrectangular (a general parallelepiped) unit cell the Green function takes a different form. However the only property we require is its periodicity. Because the periodicity of the Green function ensures a vanishing of the surface integral on the cell boundary $\partial \mathcal{C}$ which in principle should appear in the expression for the Poisson equation solution [10]. First-order variation of the expression determining the Coulomb energy

$$
\delta^{(1)} \int_{\mathcal{C}} \Phi \rho d^{3} x=\int_{\mathcal{C}} \delta^{(1)} \Phi \rho d^{3} x+\int_{\mathcal{C}} \Phi \delta^{(1)} \rho d^{3} x
$$

may be simplified by use of the reciprocity theorem (the proof is given in Appendix A)

$$
\int_{\mathcal{C}} \delta \Phi \rho d^{3} x=\int_{\mathcal{C}} \Phi \delta \rho d^{3} x
$$

Then the variation of the Coulomb energy density in the first order may be derived as the doubled charge variation times the unperturbed potential

$$
\delta^{(1)} \varepsilon_{\text {Coul }}=\frac{1}{V_{\mathcal{C}}} \int_{\mathcal{C}} \Phi \delta^{(1)} \rho d^{3} x .
$$

Equation (15) appears to be very useful-it allows to avoid the calculation of potential variation $\delta \Phi$ as long as we are interested in a first-order variation of the energy. The first-order potential perturbation will have to be calculated explicitly only in the second-order variation of energy.

The variation with respect to electron number density $d n_{e}$ corresponds to $\delta \rho(x)=d n_{e}$ for all $x \in \mathcal{C}$ whereas the proton number change $d n_{p}$ corresponds to $\delta \rho(x)=d n_{p}$ only for $x \in \mathcal{P}$. Then we get two relations, one coming from $\frac{\partial \tilde{\varepsilon}}{\partial n_{e}}=0$,

$$
\mu_{e}-\lambda_{Q}-\frac{e}{V_{\mathcal{C}}} \int_{\mathcal{C}} \Phi d^{3} x=0,
$$

and the second from $\frac{\partial \tilde{\varepsilon}}{\partial n_{p}}=0$,

$$
\frac{V_{\mathcal{P}}}{V_{\mathcal{C}}}\left(\mu_{p}-\lambda_{B}+\lambda_{Q}\right)+\frac{e}{V_{\mathcal{C}}} \int_{\mathcal{P}} \Phi d^{3} x=0 .
$$

By eliminating the Lagrange multipliers and making use of the neutrality condition $N_{e}=N_{p}$ one gets the well-known $\beta$ equilibrium condition, here, however, modified by the presence of the Coulomb energy

$$
\mu_{n}-\mu_{p}-\mu_{e}=\frac{2 E_{\mathrm{Coul}}}{N_{e}} .
$$

The Coulomb energy $E_{\text {Coul }}=\frac{1}{2} \int_{\mathcal{C}} \Phi \rho d^{3} x$ enters into the $\beta$ equilibrium condition as a consequence of long-range electrostatic interactions between protons and electrons. It is indeed the $\beta$ equilibrium condition if one only remembers that proper chemical potential $\tilde{\mu}_{i}$ includes all forms of the energy

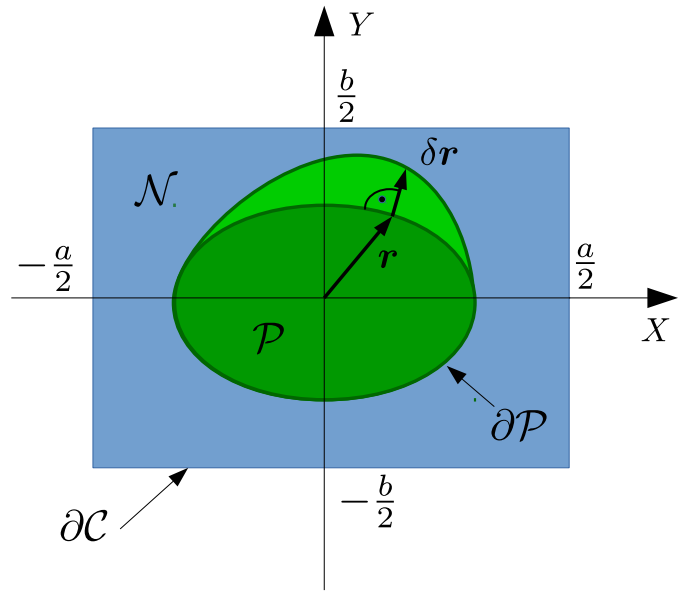

FIG. 2. The infinitesimal deformation of the proton cluster described by the normal component $\epsilon$.

concerning the $i$ particle. Then for charged particles (protons and electrons) their proper chemical potential is

$$
\tilde{\mu}_{p}=\mu_{p}+E_{p}^{(e l)}, \quad \tilde{\mu}_{e}=\mu_{p}+E_{e}^{(e l)},
$$

where $\mu_{p}, \mu_{e}$ are appropriate derivatives of $\varepsilon_{n u c}, \varepsilon_{e}$, and $E_{1}^{(e l)}$ represents the one-particle electrostatic energy. The measure of the energy is the total Coulomb energy of the cell per charged particle $\frac{E_{\text {Coul }}}{N_{p}}, \frac{E_{\text {Coul }}}{N_{e}}$. Remembering that $N_{e}=N_{p}$ one gets the standard $\beta$ equilibrium equation:

$$
\tilde{\mu}_{n}-\tilde{\mu}_{p}-\tilde{\mu}_{e}=0 .
$$

The inspection of the variation with respect to the proton cluster shape requires the methods of differential geometry $[11,12]$. Let us assume that vector valued function $\boldsymbol{r}(u, v)$ represents a parametrization of the cluster surface $\partial \mathcal{P}$. Next consider an infinitesimal deformation of $\partial \mathcal{P}$

$$
\boldsymbol{r}^{\prime}(u, v)=\boldsymbol{r}(u, v)+\delta \boldsymbol{r}(u, v) .
$$

Any changes in surface area or volume of the cluster depend only on the normal component of the $\delta \boldsymbol{r}$, Fig. 2. Then, in order to consider changes in the energy it is enough to take only its normal component of deformation, described by one scalar function $\epsilon(u, v)$ :

$$
\delta \boldsymbol{r}(u, v)=\epsilon(u, v) \boldsymbol{n},
$$

where $\boldsymbol{n}$ is an outwardly directed normal vector to the unperturbed surface. The change in the cluster shape makes the charge perturbation $\delta^{(1)} \rho$, up to the first order in the $\epsilon$ may be expressed in terms of surface $\delta$ function

$$
\delta^{(1)} \rho(\boldsymbol{x})=\Delta \rho \in \delta_{\partial \mathcal{P}}(\boldsymbol{x}),
$$

where $\Delta \rho$ is the charge contrast, i.e., $\Delta \rho=\rho_{+}-\rho_{-}=e n_{p}$. An illustrative example showing how the shape deformation leads to surface integrals is given in Appendix B. Finally, thanks to Eq. (16) and the expression for the charge perturbation, Eq. (24), the change in the Coulomb energy takes the form of surface integral

$$
\delta^{(1)} \varepsilon_{\text {Coul }}=\frac{1}{V_{\mathcal{C}}} \int_{\partial \mathcal{P}} \Phi(\boldsymbol{r}(u, v)) \Delta \rho \epsilon(u, v) d S .
$$


The change of the surface energy, in the first order of $\epsilon$, is connected to the mean curvature $H$ of cluster surface and is expressed by the integral [11]

$$
\delta^{(1)} \varepsilon_{S}=-\frac{2}{V_{\mathcal{C}}} \sigma \int_{\mathcal{P}} H(u, v) \epsilon(u, v) d S .
$$

The mean curvature $H$ is given by

$$
H=\frac{1}{2}\left(\kappa_{1}+\kappa_{2}\right) \text {, }
$$

where $\kappa_{i}$ are the principal curvatures. Here we keep the convention commonly used in mathematics that $H$ is negative for a convex surface, i.e., such surface that bends toward the region which is surrounded by the surface. That is the reason of the presence of the minus sign in Eq. (26). For example, in this convention, for a spherical proton cluster $H=-\frac{1}{R}$, for a cylindrical $H=-\frac{1}{2 R}$. As we want to find a variation of $\tilde{\varepsilon}$ we also need the variation in the cluster volume $V_{\mathcal{P}}$, which is

$$
\delta^{(1)} V_{\mathcal{P}}=\int_{\mathcal{P}} \epsilon(u, v) d S .
$$

Using Eqs. (25), (26), (28) for a functional of the energy $\tilde{\varepsilon}$ and eliminating again the Lagrange multipliers $\lambda_{B}, \lambda_{Q}$ one gets the equation for the surface curvature

$$
P^{\mathcal{P}}-P^{\mathcal{N}}=-2 \sigma H+\Delta \rho\left(\Phi-\langle\Phi\rangle_{\mathcal{P}}\right) .
$$

This equation is an extension of the Young-Laplace equation for the relation between the pressure difference [13] between the two regions separated by a layer with surface tension $\sigma$. The last term in the right-hand side (RHS) of Eq. (29) is the average of the electric potential taken on the volume of proton cluster

$$
\langle\Phi\rangle_{\mathcal{P}}=\frac{1}{V_{\mathcal{P}}} \int_{\mathcal{P}} \Phi d^{3} x
$$

Thanks to the difference $\Phi-\langle\Phi\rangle_{\mathcal{P}}$, Eq. (29) is gauge invariant, i.e., does not change its form after the transformation $\Phi \rightarrow$ $\Phi+c$, where $c$ is any constant. One should expect such behavior as the potential itself is not an observable.

Equation (29) is an equation for two unknown functions: curvature $H$ and $\Phi$, where $H$ is defined only for the surface $\mathcal{P}$, whereas the potential is defined for the whole cell and fulfills the Poisson equation (8). It implies one important conclusion that the cluster surface is not, in general, a surface with constant mean curvature (CMC surface). It is rather the surface with prescribed curvature. The surface curvature $H$ becomes a function determined by the potential $\Phi$ and pressure difference. In the commonly used Wigner-Seitz approximation for a spherical or cylindrical isolated cell the periodicity is abandoned. However in this case one may repeat the above calculations if the periodic boundary conditions are replaced by the Neumann condition, i.e., a normal derivative of the potential at the cell boundary vanishes, $\left.\partial_{n} \Phi\right|_{\mathcal{C}}=0$. Then one of the equipotential surfaces coincides with the cluster surface and in fact it is a CMC surface-sphere or cylinder. In this manner Eq. (29) reproduces the results presented earlier in $[14,15]$.

The last variation which must be considered is the change in the sizes of cell $a \rightarrow a+d a$ and similarly for the rest of walls $b$ and $c$, Fig. 3. Such an increase in cell sizes again leads

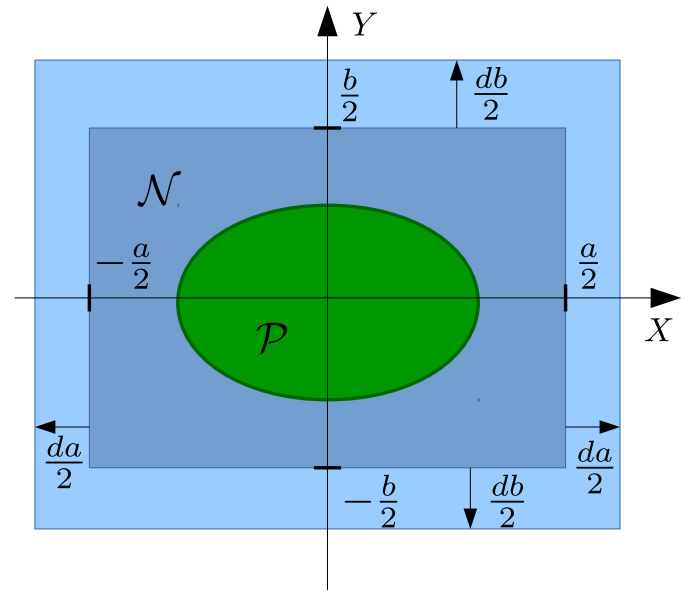

FIG. 3. Schematic view of the cell size variation.

to a change in the charge distribution. Similarly like in the case of cluster shape variation the $\delta \rho$ could be expressed by the Dirac $\delta$ function for subsequent walls:

$$
\begin{aligned}
& \delta_{a} \rho(\boldsymbol{x})=\rho_{-} \frac{d a}{2}\left(\delta\left(x-\frac{a}{2}\right)+\delta\left(x+\frac{a}{2}\right)\right), \\
& \delta_{b} \rho(\boldsymbol{x})=\rho_{-} \frac{d b}{2}\left(\delta\left(y-\frac{b}{2}\right)+\delta\left(y+\frac{b}{2}\right)\right), \\
& \delta_{c} \rho(\boldsymbol{x})=\rho_{-} \frac{d c}{2}\left(\delta\left(z-\frac{c}{2}\right)+\delta\left(z+\frac{c}{2}\right)\right) .
\end{aligned}
$$

Once more the charge variation leads to the change in the potential which can be removed by the reciprocity theorem, Eq. (15), and one gets a useful expression for the variation of Coulomb energy

$$
\delta_{a}^{(1)} E_{\mathrm{Coul}}=\frac{d a}{2} \rho_{-} \int_{ \pm a / 2} \Phi d S,
$$

where the integration is over the area of the two sides, left: $x=$ $-a / 2$ and right: $x=a / 2$ and $d S=d y d z$. The same form we get for the other two pairs of walls at $y= \pm b / 2$ and $z= \pm c / 2$. By writing down the variation of $\tilde{\varepsilon}$ with respect to $d a, d b, d c$ and eliminating the Lagrange multipliers one gets the three equations

$$
\begin{aligned}
& 2 V_{\mathcal{P}} \sigma H+E_{s}=-E_{\mathrm{Coul}}+\left.e N_{e} \Phi\right|_{\partial \mathcal{P}}-\frac{e N_{e} a}{2 V_{\mathcal{C}}} \int_{ \pm a / 2} \Phi d S, \\
& 2 V_{\mathcal{P}} \sigma H+E_{s}=-E_{\mathrm{Coul}}+\left.e N_{e} \Phi\right|_{\partial \mathcal{P}}-\frac{e N_{e} b}{2 V_{\mathcal{C}}} \int_{ \pm b / 2} \Phi d S, \\
& 2 V_{\mathcal{P}} \sigma H+E_{s}=-E_{\mathrm{Coul}}+\left.e N_{e} \Phi\right|_{\partial \mathcal{P}}-\frac{e N_{e} c}{2 V_{\mathcal{C}}} \int_{ \pm c / 2} \Phi d S .
\end{aligned}
$$

Those three equations are not independent. Equations (35) include integrals which are surface integral only on pairs of the opposite sides of the cell. By taking the linear combination of them we may get more a compact relation

$$
2 V_{\mathcal{P}} \sigma H+E_{s}=-E_{\mathrm{Coul}}+e N_{e}\left(\left.\Phi\right|_{\partial \mathcal{P}}-\langle\Phi\rangle_{\partial \mathcal{C}}\right),
$$


where the last term in the RHS is the average of the potential taken on the whole boundary of the cell

$$
\langle\Phi\rangle_{\partial \mathcal{C}}=\frac{1}{2(a b+a c+b c)} \int_{\partial \mathcal{C}} \Phi d S .
$$

Equation (36) represents a generalization of the so-called "virial" theorem known from previous works $[1,14]$ where the relation between $E_{s}$ and $E_{c}$ was shown for an isolated Wigner-Seitz cell. For such kinds of cell which are constructed without periodic boundary conditions Eq. (36) may still be used. This comes from the fact that such an isolated cell, when neutral, obeys the Neumann boundary condition, $\left.\frac{\partial \Phi}{\partial n}\right|_{\partial \mathcal{C}}=0$, and then all of our calculations may be repeated. The crucial point is the reciprocity theorem, Eq. (15), which is valid both for the periodic potential and for the potential with Neumann BC (see Appendix A). It may easily checked that Eq. (36) for the simplified geometry of pastas (gnocchi, spaghetti, lasagna) takes the form

$$
E_{s}=2 E_{\text {Coul }} .
$$

However, one must remember that Eq. (38) is fulfilled only in cases with such high symmetry (spheres, cylinders, or slabs) and in general do not need to be true and the general virial theorem is represented by Eq. (36).

\section{LIMITED STABILITY CONSIDERATION}

The set of equations (10), (19), (29), (35) comes form the first-order analysis and represents the necessary condition for a minimum of the energy. Equations (10), (19) correspond to chemical equilibrium, Eq. (29) represents the extension of the Young-Laplace equation for charged fluid, and Eqs. (35) stand for the generalization of the virial theorem. In the next step it would be desirable to test the energy change up to the second order of all relevant variables, that means densities $n_{p}, n_{e}, n_{n}^{\mathcal{P}}, n_{n}^{\mathcal{N}}$, cell sizes $a, b, c$, and the change of the cluster shape $\epsilon(u, v)$. For that reason the second-order variation of energy $\tilde{\varepsilon}$ would be an $8 \times 8$ matrix. Here we constrain only to the analysis with respect to the shape variation $\epsilon$. It does not present the full analysis of stability, nevertheless we would like to show it as it is the most complicated part of the second-order energy variation and leads to an interesting result. Physically, such analysis corresponds to testing the shape stability when all particle densities and cell sizes are kept constant.

The variation of the surface energy with respect to the second order in the shape change $\epsilon(u, v)$ is expressed by the integral over the cluster surface [11]

$$
\delta^{(2)} \varepsilon_{S}=\frac{\sigma}{2 V_{\mathcal{C}}} \int_{\partial \mathcal{P}}\left((\nabla \epsilon)^{2}+2 K \epsilon^{2}\right) d S,
$$

where $K$ is the Gauss curvature $K=\kappa_{1} \kappa_{2}$. The second-order change in the volume of the cluster takes the form

$$
\delta^{(2)} V_{\mathcal{P}}=-\int_{\partial \mathcal{P}} H \epsilon^{2} d S .
$$

In order to find the variation of the Coulomb energy we must know the charge distribution variation caused by the change in the proton cluster up to the second order in normal deformation $\epsilon$. It is convenient to describe the charge variation by the surface $\delta$ function [12]

$$
\delta^{(2)} \rho(\boldsymbol{x})=\frac{1}{2} \Delta \rho \epsilon^{2}\left(\partial_{n}-2 H\right) \delta_{\partial \mathcal{P}}(\boldsymbol{x}),
$$

where $\partial_{n}=\boldsymbol{n} \circ \nabla$ is the normal derivative operator acting on the potential $\Phi$ at the cluster surface $\partial \mathcal{P}$.

The second-order variation of the Coulomb energy depends not only on the $\delta \rho$ and $\delta \Phi$ in second order but also on the first-order variation of potential and charge distribution

$$
\delta^{(2)} \varepsilon_{\text {Coul }}=\frac{1}{2 V_{\mathcal{C}}} \int_{\mathcal{C}}\left(\delta^{(2)} \Phi \rho+\Phi \delta^{(2)} \rho+\delta^{(1)} \Phi \delta^{(1)} \rho\right) d^{3} x .
$$

We do not need to calculate the second-order variation of the potential $\delta^{(2)} \Phi$, as it may be removed by use of reciprocity theorem

$$
\int_{\mathcal{C}} \delta^{(2)} \Phi \rho d^{3} x=\int_{\mathcal{C}} \Phi \delta^{(2)} \rho d^{3} x .
$$

The last term in Eq. (42) requires a derivation of the first-order variation of the potential $\delta^{(1)} \Phi$ :

$$
\delta^{(1)} \Phi(\boldsymbol{x})=\int_{\mathcal{C}} G_{P}\left(\boldsymbol{x}, \boldsymbol{x}^{\prime}\right) \delta^{(1)} \rho\left(\boldsymbol{x}^{\prime}\right) d^{3} x^{\prime} .
$$

Using the above expressions for the charge and potential variation one gets the second-order variation of the Coulomb energy

$$
\delta^{(2)} \varepsilon_{\text {Coul }}=\frac{1}{2 V_{\mathcal{C}}} \int_{\partial \mathcal{P}}\left(\Delta \rho\left(\partial_{n} \Phi-2 H \Phi\right) \epsilon+\delta_{\epsilon} \Phi\right) \epsilon d S,
$$

where we denoted the first-order variation of the potential by $\delta_{\epsilon} \Phi(\boldsymbol{x})$,

$$
\delta_{\epsilon} \Phi(\boldsymbol{x})=\delta^{(1)} \Phi(\boldsymbol{x})=\int_{\partial \mathcal{P}} \Delta \rho G_{P}\left(\boldsymbol{x}, \boldsymbol{x}^{\prime}\right) \epsilon\left(\boldsymbol{x}^{\prime}\right) d S^{\prime},
$$

to indicate it is the linear functional of deformation $\epsilon$. Collecting together all parts of $\tilde{\varepsilon}$ and eliminating Lagrange multipliers by use of the equation coming from the first-order analysis, Eqs. (10), (17), (18) we get the second-order variation of the constrained energy

$$
\begin{aligned}
\delta^{(2)} \tilde{\varepsilon}= & \frac{1}{2} \int_{\partial \mathcal{P}}\left(\sigma\left((\nabla \epsilon)^{2}-B^{2} \epsilon^{2}\right)\right. \\
& \left.+\Delta \rho\left(\partial_{n} \Phi \epsilon^{2}+\delta_{\epsilon} \Phi \epsilon\right)\right) d S,
\end{aligned}
$$

where $B^{2}$ is the sum of squared principal curvatures

$$
B^{2}=4 H^{2}-2 K=\kappa_{1}^{2}+\kappa_{2}^{2} .
$$

The positivity of $\delta^{(2)} \tilde{\varepsilon}$ stands for the sufficient condition for the minimum of total energy with imposed constraints, Eqs. (2), (3). However one must remember that $\delta^{(2)} \tilde{\varepsilon}$ must be positive not for any shape variation but for a variation consistent with the constraints. Here it means we may take into account only the volume-preserving variation. Finally, the stability condition 
states

$$
\begin{aligned}
& \sigma\left((\nabla \epsilon)^{2}-B^{2} \epsilon^{2}\right)+\Delta \rho\left(\partial_{n} \Phi \epsilon^{2}+\delta_{\epsilon} \Phi \epsilon\right)>0 \\
& \text { for any } \epsilon \text { such that } \int_{\partial \mathcal{P}} \epsilon d S=0 .
\end{aligned}
$$

Let us comment on the subsequent parts of the $\delta^{(2)} \tilde{\varepsilon}$. The squared deformation gradient $(\nabla \epsilon)^{2}$ gives an always positive contribution as it corresponds to the increase of the surface area caused by deformation. Always negative, the curvature term $-B^{2} \epsilon^{2}$ pictures the tendency of the surface to contract locally. The competition of these two terms leads to the well-known Rayleigh-Plateau instability for a cylindrical spurt of fluid [16]. The two other terms come from the presence of charged phases in the system. The first one of them, $\partial_{n} \Phi \epsilon^{2}$, is always negative, because $\partial_{n} \Phi=-\boldsymbol{n} \circ \boldsymbol{E}$ and the electric field $\boldsymbol{E}$ for a positively charged cluster is directed outward, like the normal vector $\boldsymbol{n}$. For the last term, the potential variation $\delta_{\epsilon} \Phi$, it is difficult to determine its sign. Then we cannot say clearly what its effect is on the stability. From that it is an open question whether the presence of charge in the system makes the cluster stable or not.

The second-order variation allows to find an equation, being the condition for breaking the stability. The divergence theorem applied to $\epsilon$ on the cluster surface [11] allows to replace the squared gradient of $\epsilon$ by its Laplacian. So, we get the expression for the second-order variation of $\tilde{\varepsilon}$ :

$$
\delta^{(2)} \tilde{\varepsilon}=\frac{1}{2} \int_{\partial \mathcal{P}}\left(-\sigma\left(\nabla^{2} \epsilon+B^{2} \epsilon\right)+\Delta \rho\left(\partial_{n} \Phi \epsilon+\delta_{\epsilon} \Phi\right)\right) \epsilon d S .
$$

If there exist any $\epsilon$ making the cluster unstable, then the energy variation has to change its sign and the following equation must be fulfilled:

$$
-\sigma\left(\nabla^{2} \epsilon+B^{2} \epsilon\right)+\Delta \rho\left(\partial_{n} \Phi \epsilon+\delta_{\epsilon} \Phi\right)=0 .
$$

In the absence of electrostatic interactions $(\Delta \rho \rightarrow 0)$ the above equation becomes the well-known Jacobi equation

$$
\nabla^{2} \epsilon+B^{2} \epsilon=0
$$

appearing in the stability analysis of surface with a given curvature [11]. Equation (51) represents a generalization of the Jacobi equation in the presence of electrostatic forces. One must mention that by the potential variation $\delta_{\epsilon} \Phi$, given by the integral Eq. (46), now, the extended Jacobi equation becomes an integrodifferential equation.

The stability condition in the form of Eq. (49) appears to be quite general. It may be also applied to the stability consideration for the liquid drop model of an isolated nucleus. The only difference in comparison to our analysis is that instead of the periodic Green function one should use the vacuum Green function $G_{\mathrm{vac}}\left(\boldsymbol{x}, \boldsymbol{x}^{\prime}\right)=1 /\left|\boldsymbol{x}-\boldsymbol{x}^{\prime}\right|$. The crucial point is that for potential variation the reciprocity theorem is still valid. Then the whole previous considerations could be applied also in this case.

Let us consider the spherically symmetric nucleus with charge $Z e$, radius $R$, and surface tension $\sigma$. The quadrupole deformation in spherical coordinates takes the form

$$
\epsilon(\theta, \phi)=\epsilon P_{2}(\cos \theta) .
$$

Here, $\epsilon$ is any number and $P_{2}$ is Legendre polynomial. Such deformation preserves volume in the first order as it is required in Eq. (49). The charge contrast is $\Delta \rho=\frac{3 Z e}{4 \pi R^{3}}$ and subsequent terms are $B^{2}=2 / R^{2}, \partial_{n} \Phi=-Z e / R^{2}$, and $\delta_{\epsilon} \Phi=\frac{4 \pi}{5} \Delta \rho R \epsilon$. Putting those terms into Eq. (49) and remembering that nucleus radius scales with mass number as $R=r_{0} A^{1 / 3}$, one gets the well-known Bohr-Wheeler condition for nuclear fission by quadruple deformation of nucleus [17]

$$
\frac{Z^{2}}{A}>\frac{40 \pi \sigma r_{0}^{3}}{3 e^{2}} \text {. }
$$

\section{SUMMARY}

In this work, for the first time, the equilibrium equations for pasta phases were derived in a more rigorous treatment. We abandon the idea of an isolated Wigner-Seitz cell. Assuming that pastas should form periodic structures, the periodic boundary conditions were imposed without any assumptions on the geometry of the phases. The most relevant conclusion is that the shape of the proton cluster $\mathcal{P}$ is determined by the pair of equations

$$
\begin{aligned}
P^{\mathcal{P}}-P^{\mathcal{N}} & =-2 \sigma H+\Delta \rho\left(\Phi-\langle\Phi\rangle_{\mathcal{P}}\right), \\
\nabla^{2} \Phi(\boldsymbol{x}) & =-4 \pi \rho(\boldsymbol{x}) .
\end{aligned}
$$

The first is the generalized Young-Laplace equation which connects the mean curvature $H(x), x \in \partial \mathcal{P}$ with periodic electrostatic potential $\Phi(\boldsymbol{x})$. The potential itself depends on the cluster shape, as the charge distribution is determined by the cluster surface. Now, the cluster surface belongs to the class of surfaces with prescribed mean curvature

$$
H(\boldsymbol{x})=C+f(\boldsymbol{x}),
$$

where $C$ is a constant and $f(\boldsymbol{x})$ is scaled electrostatic potential.

Finding the surface with the prescribed curvature presents a highly nonlinear problem. Some general results based on complex analysis are known [18] but in our case numerical solving of the equations seems to be unavoidable. Various numerical methods like the finite-element [19] or level-set approach [20] could be applied. As the aim of this work was to present the mathematically rigorous approach to periodic structures of pasta phases the concrete results coming from numerical calculations are going to be presented in a future work.

We also generalized other equations governing the state of the charged two-phase system. The $\beta$ equilibrium

$$
\mu_{n}-\mu_{p}-\mu_{e}=\frac{2 E_{\mathrm{Coul}}}{N_{e}}
$$

and virial theorem

$$
2 V_{\mathcal{P}} \sigma H+E_{s}=-E_{\text {Coul }}+e N_{e}\left(\left.\Phi\right|_{\partial \mathcal{P}}-\langle\Phi\rangle_{\partial \mathcal{C}}\right) .
$$

Stability of pasta phases was never considered, up to now. The idea of an isolated cell in the Wigner-Seitz approximation strongly limits the inspection of this issue. Introduction of a unit cell with periodic boundary conditions makes stability 
analysis more reliable. The full stability analysis would require testing the second-order variation of energy with respect to all variables. In this work we restrict ourselves to the most interesting one-the cluster shape changes. As a result we obtained an extension of the Jacobi equation

$$
-\sigma\left(\nabla^{2} \epsilon+B^{2} \epsilon\right)+\Delta \rho\left(\partial_{n} \Phi \epsilon+\delta_{\epsilon} \Phi\right)=0
$$

which allows for seeking the deformation $\epsilon$ which breaks the stability.

The work opens new ways to a better understanding and analysis of properties of pasta phases appearing in neutron stars. It is worth it to mention the results presented here use general conceptions of charged phases with surface tension and as such could be also relevant for any system containing charged fluid like in the electrowetting phenomena [21].

\section{APPENDIX A: RECIPROCITY THEOREM}

Here we show the reciprocity theorem in the case of periodic potential and its variation. Let us write down the Green identity for $\Phi$ and $\delta \Phi$ :

$\int_{\mathcal{C}}\left(\Phi \nabla^{2} \delta \Phi-\delta \Phi \nabla^{2} \Phi\right) d^{3} x=\int_{\partial \mathcal{C}}\left(\Phi \partial_{n} \delta \Phi-\delta \Phi \partial_{n} \Phi\right) d S$.

Both the potential and its variation are solutions of the periodic Poisson equation

$$
-4 \pi \int_{\mathcal{C}}(\Phi \delta \rho-\delta \Phi \rho) d^{3} x=\int_{\partial \mathcal{C}}\left(\Phi \partial_{n} \delta \Phi-\delta \Phi \partial_{n} \Phi\right) d S .
$$

The left-hand side (LHS) of the above equation is 0 because the contribution from the normal derivative of $\Phi$ or $\delta \Phi$ taken for opposite sides of the cell are canceled, for example for the side perpendicular to the $x$ axis

$$
\left.\partial_{n} \Phi\right|_{x=-a / 2}=-\left.\partial_{n} \Phi\right|_{x=a / 2}
$$

and similarly for the other cell sides. In the case of an isolated cell (sphere, cylinder) instead of periodic boundary conditions the potential and its variation obeys the Neumann boundary condition

$$
\left.\partial_{n} \Phi\right|_{\partial \mathcal{C}}=0
$$

and again the LHS of Eq. (A1) vanishes.

\section{APPENDIX B: SHAPE DEFORMATION AND SURFACE INTEGRALS}

Here we illustrate how the charge variation $\delta \rho$ caused by the change in cluster shape $\epsilon$ may be expressed by the surface integral on an unperturbed cluster surface. As an example let us consider a cylindrical cluster with radius $R$ perturbed by a deformation $\epsilon$, see Fig. 4. The deformation $\epsilon(z, \phi)$ is a function defined on the cylinder surface $r=R$.

In cylindrical coordinates $r, \phi, z$ the charge distribution, before and after deformation, is given by

$$
\begin{gathered}
\rho(r, \phi, z)=\rho_{-}+\left(\rho_{+}-\rho_{-}\right) \theta(R-r), \\
\rho^{\prime}(r, \phi, z)=\rho_{-}+\left(\rho_{+}-\rho_{-}\right) \theta(R+\epsilon-r) .
\end{gathered}
$$
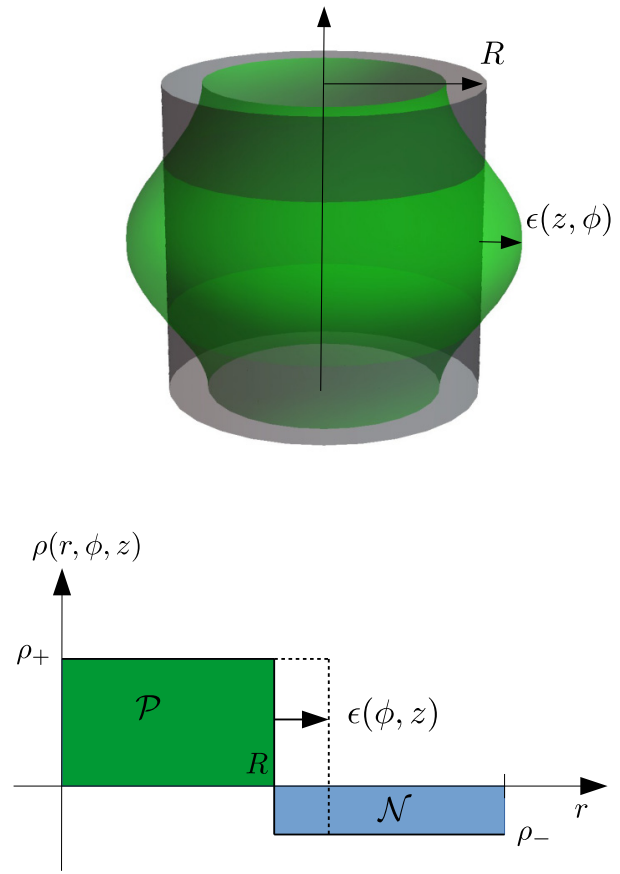

FIG. 4. (a) The axially symmetric deformation of cylindrical cluster. (b) shows the charge density profile for a fixed $\phi, z$.

By use of the Taylor expansion in powers of $\epsilon(z, \phi)$ one gets

$\delta \rho(r, \phi, z)=\Delta \rho \delta(R-r) \epsilon(z, \phi)-\frac{1}{2} \Delta \rho \delta^{\prime}(R-r) \epsilon(z, \phi)^{2}$.

The expression for electrostatic energy or perturbed potential is always represented by a linear functional of $\delta \rho$ integrated with some function $f$ [Eqs. (16), (42), (44)], which in our example appears to be

$$
\begin{aligned}
\int_{\mathcal{C}} f & \delta \rho d^{3} x \\
= & \Delta \rho \int_{S} f(R, z, \phi) \epsilon(z, \phi) R d \phi d z \\
& +\frac{\Delta \rho}{2} \int_{S}\left(\frac{\partial f}{\partial r}(R, z, \phi)+\frac{1}{R} f(R, z, \phi)\right) \epsilon(z, \phi)^{2} R d \phi d z .
\end{aligned}
$$

One may notice that in the second order, the integration of $\delta^{\prime}(r-R)$ with the volume element $r d r d \phi d z$ introduces the doubled cylinder curvature $\left(H=-\frac{1}{2 R}\right)$. For the strict proof see [12]. The resulting surface integral may again be written down as the volume integral if one uses the surface $\delta$ function $\delta_{S}(\boldsymbol{x})$ which is defined by the property

$$
\int_{\mathbf{R}^{3}} f(\boldsymbol{x}) \delta_{S}(\boldsymbol{x}) d^{3} x=\int_{S} f d S,
$$

where $S$ is any surface inside the three-dimensional space. In this way it appears to be convenient to represent the charge perturbation $\delta \rho$ in terms of the surface $\delta$ function as in formulas (24), (41). 
[1] G. Baym, H. A. Bethe, and C. Pethick, Nucl. Phys. A 175, 225 (1971).

[2] D. G. Ravenhall, C. J. Pethick, and J. R. Wilson, Phys. Rev. Lett. 50, 2066 (1983).

[3] M. Hashimoto, H. Seki, and M. Yamada, Prog. Theor. Phys. 71, 320 (1984).

[4] C. J. Pethick and D. G. Ravenhall, Annu. Rev. Nucl. Part. Sci. 45, 429 (1995).

[5] K. Nakazato, K. Oyamatsu, and S. Yamada, Phys. Rev. Lett. 103, 132501 (2009).

[6] R. D. Williams and S. E. Koonin, Nucl. Phys. A 435, 844 (1985).

[7] B. Schuetrumpf, M. A. Klatt, K. Iida, G. E. Schroder-Turk, J. A. Maruhn, K. Mecke, and P.-G. Reinhard, Phys. Rev. C 91, 025801 (2015).

[8] A. S. Schneider, C. J. Horowitz, J. Hughto, and D. K. Berry, Phys. Rev. C 88, 065807 (2013).

[9] S. L. Marshall, J. Phys.: Condens. Matter 12, 4575 (2000); S. Tyagi, Phys. Rev. E 70, 066703 (2004).

[10] J. D. Jackson, Classical Electrodynamics (John Wiley \& Sons, New York, 1999).
[11] K. Grosse-Brauckmann, Lectures on surfaces of constant mean curvature https://www3.mathematik.tu-darmstadt.de/evs/e/ 32.html? evsver $=867 \&$ evsdir $=819 \&$ evsfile $=$ cmc.pdf.

[12] V. A. Klyachin, Izv. Math. 70, 717 (2006).

[13] J. Berthier and K. A. Brakke, The Physics of Microdroplets (John Wiley \& Sons, New York, 2012).

[14] P. Haensel, A. Y. Potekhin, and D. G. Yakovlev, Neutron Stars 1: Equation of State and Structure (Springer, Berlin, 2007).

[15] H. Pais, S. Chiacchiera, and C. Providencia, Phys. Rev. C 91, 055801 (2015).

[16] Strutt, J. W. Lord Rayleigh, Proc. London Math. Soc. 10, 4 (1878).

[17] N. Bohr and J. A. Wheeler, Phys. Rev. 56, 426 (1939).

[18] K. Kenmotsu, Math. Ann. 245, 89 (1979); Tohoku Math. Journ. 32, 147 (1980).

[19] G. Dziuk and J. Hutchinson, J. Numer. Math. 102, 611 (2006).

[20] S. Osher and J. A. Sethian, J. Comput. Phys. 79, 12 (1988).

[21] L. Chen and E. Bonaccurso, Adv. Colloid Interface Sci. 210, 2 (2014). 